\title{
Effects of social diversity on the emergence of global consensus in opinion dynamics
}

\author{
Han-Xin Yang, ${ }^{1,2, *}$ Zhi-Xi Wu, ${ }^{3}$ Changsong Zhou, ${ }^{2,4}$ Tao Zhou, ${ }^{1,5}$ and Bing-Hong Wang ${ }^{1,6, \dagger}$ \\ ${ }^{1}$ Department of Modern Physics, University of Science and Technology of China, Hefei 230026, China \\ ${ }^{2}$ Department of Physics, Hong Kong Baptist University, Kowloon Tong, Hong Kong \\ ${ }^{3}$ Department of Physics, Umeå University, 90187 Umeå, Sweden \\ ${ }^{4}$ Centre for Nonlinear Studies and The Beijing-Hong Kong-Singapore Joint Centre for Nonlinear and Complex Systems (Hong Kong), \\ Hong Kong Baptist University, Kowloon Tong, Hong Kong \\ ${ }^{5}$ Department of Physics, University of Fribourg, Chemin du Muse 3, CH-1700 Fribourg, Switzerland \\ ${ }^{6}$ The Research Center for Complex System Science, University of Shanghai for Science and Technology and Shanghai Academy \\ of System Science, Shanghai 200093, China
}

\begin{abstract}
We propose a variant of the voter model by introducing the social diversity in the evolution process. Each individual is assigned a weight that is proportional to the power of its degree, where the power exponent $\alpha$ is an adjustable parameter that controls the level of diversity among individuals in the network. At each time step, a pair of connected individuals, say $i$ and $j$, are randomly selected to update their opinions. The probability $p_{i}$ of choosing is opinion as their common opinion is proportional to is weight. We consider the scale-free topology and concentrate on the efficiency of reaching the final consensus, which is significant in characterizing the self-organized systems. Interestingly, it is found that there exists an optimal value of $\alpha$, leading to the shortest consensus time. This phenomenon indicates that, although a strong influence of high-degree individuals is helpful for quick consensus achievement, over strong influence inhibits the convergence process. Other quantities, such as the probability of an individual's initial opinion becomes the final opinion as a function of degree, the evolution of the number of opinion clusters, as well as the relationship between average consensus time and the network size, are also studied. Our results are helpful for better understanding the role of degree heterogeneity of the individuals in the opinion dynamics.
\end{abstract}

\section{INTRODUCTION}

In recent years, a large class of interdisciplinary problems has been successfully studied with statistical physics methods, in particular those related to the characterization of the collective social behavior of individuals, such as opinion formation $[1,2]$, the spreading of rumor or disease [3-6], the language dynamics [7], etc. The study of opinion dynamics has attracted much attention in the physics community and many models have been proposed to describe the processes of opinion formation [8,9], such as the majority rule model [10-12], the voter model (VM) [13], and the boundedconfidence model [14], etc. Given the recent widespread interest in complex networks $[15,16]$, dynamical progresses described by these models have been extensively studied on various networks [17-34]. Some models of opinion formation display a disorder-order transition [17,18,24], from a regime in which opinions are arbitrarily diverse to one in which most individuals hold the same opinion. Other models focus the emergence of a global consensus, in which all agents share the same opinion [11,21].

Diversity in wealth and social status is present not only among human but also throughout the animal world. Due to the existence of social diversity, the impacts of different individuals are different. For example, leaders may have stronger influence than others and their opinions may be followed

\footnotetext{
*hxyang@mail.ustc.edu.cn

†bhwang@ustc.edu.cn
}

more frequently. It has been shown that the social diversity is an important factor in opinion $[35,36]$ or other dynamics [37-39]. In Ref. [35], Galam considered heterogeneous beliefs in the making of public opinions. In Ref. [36], Guan et al. studied the effects of inhomogeneous influence of individuals on an order-disorder transition in opinion dynamics.

In this paper, we explore how the social diversity affects the emergence of global consensus in opinion dynamics. For this purpose, a variant of the voter model will be considered. Let us briefly review the VM [13]. The VM evolution consists of sequentially randomly choosing a voter (one node) who will adopt the state of a random neighbor. In our modified VM, the two selected neighboring individuals compete for becoming the dominator and the individual with stronger social influence has larger probability to impose its opinion to the other individual.

The paper is organized as follows. In Sec. II, the modified voter model is described. Results and analysis are introduced in Sec. III. The paper is concluded in Sec. IV.

\section{MODEL}

Initially, each individual holds one of $G(\geq 2)$ opinions with equal probability. $G$ is set to 2 in the original VM, however, the optional choices are usually more than 2 in many realistic situations, such as the selection of city for Olympic games, etc. For this reason, in the present study we relax the value of $G$ to arbitrary integers. Each individual $i$ is assigned a weight $k_{i}^{\alpha}$, where $k_{i}$ is the degree of $i$ and $\alpha$ is an adjustable parameter, named the diversity parameter. Here 


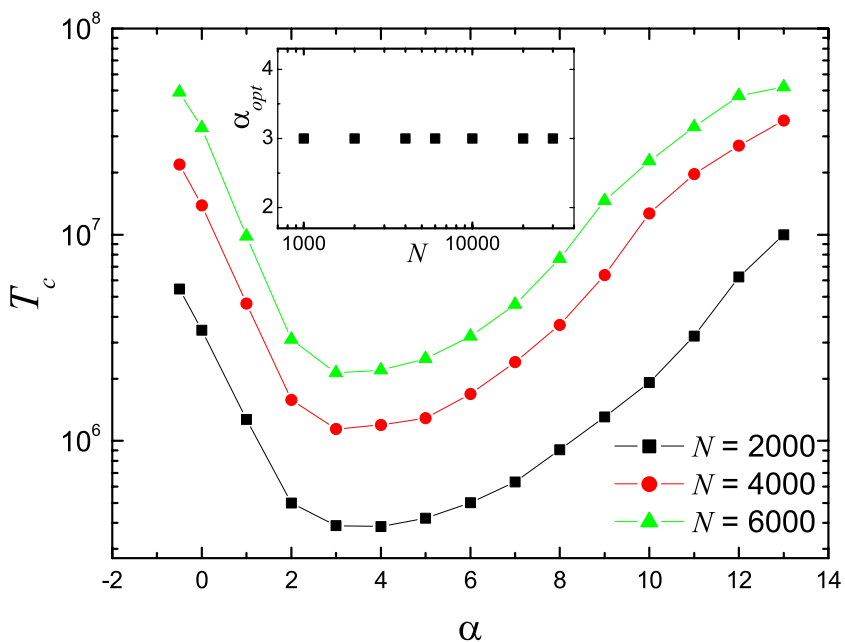

FIG. 1. (Color online) Consensus time $T_{c}$ as a function of $\alpha$ for different network size $N$. The inset shows the optimal values $\alpha_{\text {opt }}$ as a function of $N$. The average connectivity of the BA network $\langle k\rangle$ $=6$ and the number of initial opinions $G=2$. Each data point is obtained by averaging over 50 different network realizations with 50 runs for each realization.

the weight can be considered as the strength of social influence. At each time step, a pair of connected individuals are randomly selected to update their opinions. The two individuals, say $i$ and $j$, will adopt one of their opinions as the common opinion. The probability $p_{i}$ of choosing is opinion as the common opinion is proportional to $i$ s weight:

$$
p_{i}=\frac{k_{i}^{\alpha}}{k_{i}^{\alpha}+k_{j}^{\alpha}} .
$$

If $\alpha>0(<0)$, the higher-degree (lower-degree) individual has larger weight of social influence and its opinion therefore has larger probability to be selected as the common opinion. In the case of $\alpha=0$, two selected individuals' opinions have the equal probability to become the common opinion.

\section{RESULTS AND ANALYSIS}

In the following simulations, we assume that individuals are situated on nodes of a Barabási-Albert (BA) scale-free network [40] without special mention. The BA network model can be constructed as follows. Starting from $m_{0}$ nodes, a new node with $m$ edges is added at each time and preferentially attached to $m$ existing nodes with probability proportional to the degrees of existing nodes. The average connectivity of the network $\langle k\rangle=2 m$ and the degree distribution obey a power-law form $n_{k} \propto k^{-\gamma}$, with the exponent $\gamma=3$.

The efficiency of reaching the final consensus can be measured by the consensus time. Figures 1-3 show the consensus time $T_{c}$ as a function of $\alpha$ for different network size $N$, the number of initial opinions $G$, and the average connectivity $\langle k\rangle$. One can see that there exists an optimal value of $\alpha$, hereafter denoted by $\alpha_{\text {opt }}$, resulting in the shortest consensus time. Figure 1 shows that $T_{c}$ increases as the network size $N$ increases, while $\alpha_{\text {opt }}$ seems to be independent of $N$. Figure 2 shows that $T_{c}$ increases very slowly as the number of initial

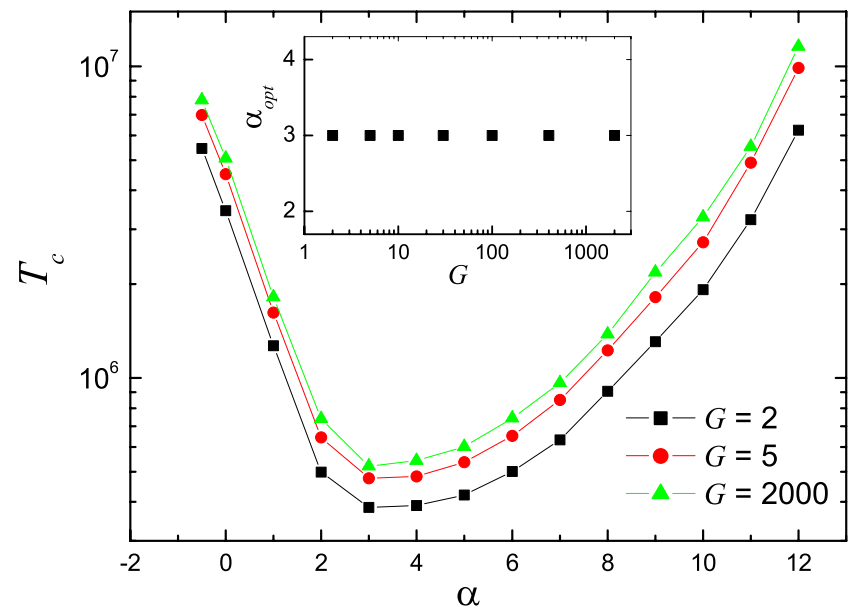

FIG. 2. (Color online) Consensus time $T_{c}$ as a function of $\alpha$ for different number of initial opinions $G$. The inset shows the optimal values $\alpha_{\text {opt }}$ as a function of $G$. The average connectivity of the BA network $\langle k\rangle=6$ and the network size $N=2000$. Each data point is obtained by averaging over 50 different network realizations with 50 runs for each realization.

opinions $G$ increases and $\alpha_{o p t}$ is found to be independent of $G$. Figure 3 displays that $T_{c}$ decreases as the average connectivity $\langle k\rangle$ increases and $\alpha_{\text {opt }}$ increases as $\langle k\rangle$ increases. Moreover, from Figs. 1-3, one can observe that the value of $\alpha_{\text {opt }}$ is positive and no less than 2 , indicating that strong influence of the high-degree individuals (but only to an appropriate extent) can best favor the achievement of consensus.

According to Eq. (1), for $\alpha$ greater than zero, the higherdegree individual's opinion is more likely to be chosen as the common opinion. Hence as $\alpha$ grows, the opinions held by the higher-degree individuals are easier to spread in the network. In fact, one can see in Fig. 4 that the initial opinions of higher-degree individuals have larger probability to become the final opinion as $\alpha$ increases. Besides, the average degree

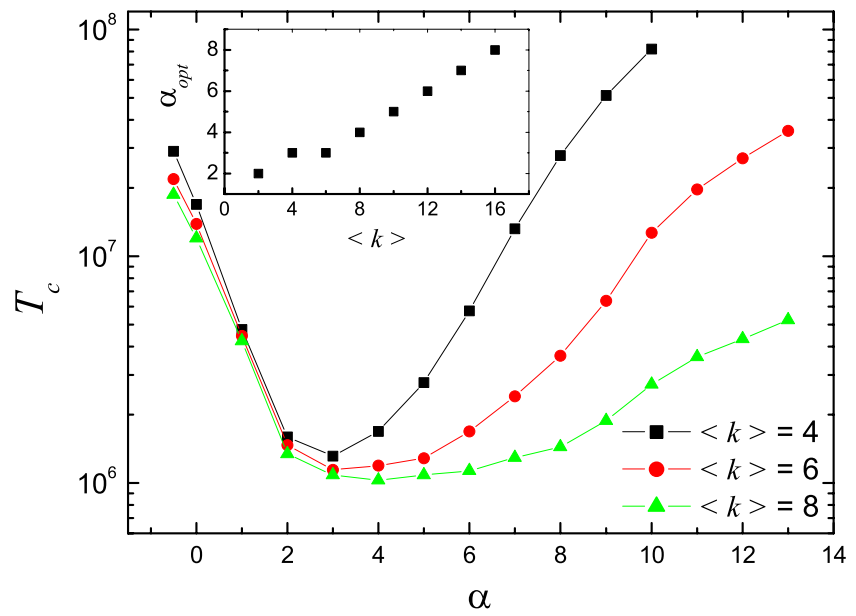

FIG. 3. (Color online) Consensus time $T_{c}$ as a function of $\alpha$ for different average connectivity $\langle k\rangle$. The inset shows the optimal values $\alpha_{\text {opt }}$ as a function of $\langle k\rangle$. The BA network size $N=4000$ and the number of initial opinions $G=2$. Each data point is obtained by averaging over 50 different network realizations with 50 runs for each realization. 


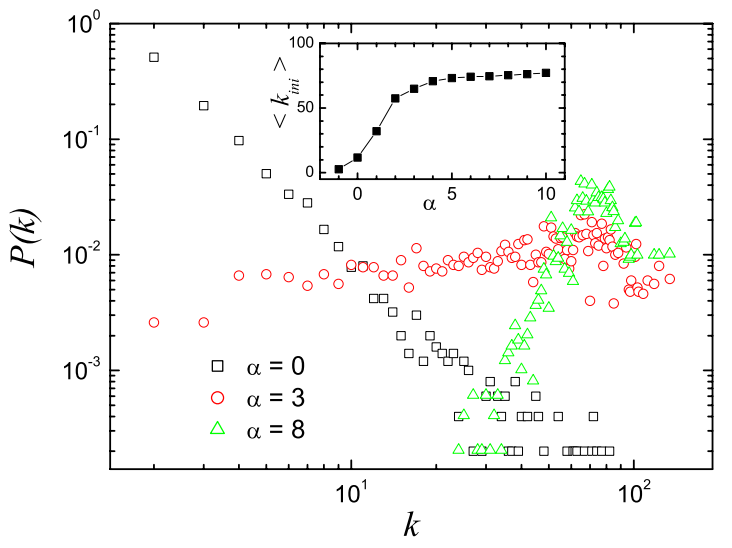

FIG. 4. (Color online) The probability $P(k)$ of an individualwhose initial opinion becomes the final opinion-as a function of degree $k$ for different values of $\alpha$. The inset shows the dependence of the average degree $\left\langle k_{\text {ini }}\right\rangle$ of individuals - whose initial opinions become the final opinion-on $\alpha$. The BA network size $N=1000$, the average connectivity $\langle k\rangle=4$, and the number of initial opinions $G$ $=N=1000$. The data are obtained by averaging over 100 different network realizations with 50 runs for each realization.

of individuals whose initial opinions become the final opinion also increases as $\alpha$ increases. In scale-free networks a few nodes have high degrees, while most nodes are of low degrees. The opinion dynamics is proceeded by formation of some big opinion clusters around the high-degree individuals, and within each cluster the individuals share a common opinion. Through the competition of different opinion clusters, one big cluster will invade the others and finally dominate the system with a global consensus. Note that the core of a cluster usually is a high-degree individual, for very high value of $\alpha$, the cluster becomes very steady due to the very strong influence of the core. As a result, the merging of different clusters become very difficult for too large $\alpha$, leading to longer consensus time. Taken together, we can expect that the shortest consensus time would be realized at moderate value of $\alpha$. This is what we have observed in Figs. 1-3.

Figure 5 shows the evolution of the number of opinion clusters $N_{c l}$ for different values of $\alpha$. One can see that as the time evolves, $N_{c l}$ eventually decreases to 1 . For $\alpha=0, N_{c l}$ decreases much more slowly, compared with $\alpha=3$ and 10, indicating that it is hard to form big opinion clusters when $\alpha$ is small. For $\alpha=10, N_{c l}$ decreases a little faster than the case of $\alpha=3$ in the early stage. However, when only a few clusters remain in the system, for example, $N_{c l}<10$, the system takes much longer time to reach the final consensus than that of $\alpha=3$, indicating that the competition among big opinion clusters becomes furious.

Then we investigate the scaling behavior of the consensus time with the network size for different values of $\alpha$. As shown in Fig. $6, T_{c}$ scales as $N^{\beta}$ with $\beta$ depending on the value of $\alpha$. In particular, the optimal $\alpha=3$ results in the lowest value of $\beta$, corroborating our previous findings.

In the above simulations, we consider the modified VM on BA scale-free networks. Note that the exponent of the power-law degree distribution is fixed at 3 for BA networks. While many real networks display different values of the exponent [15]. To understand how the exponent of the degree

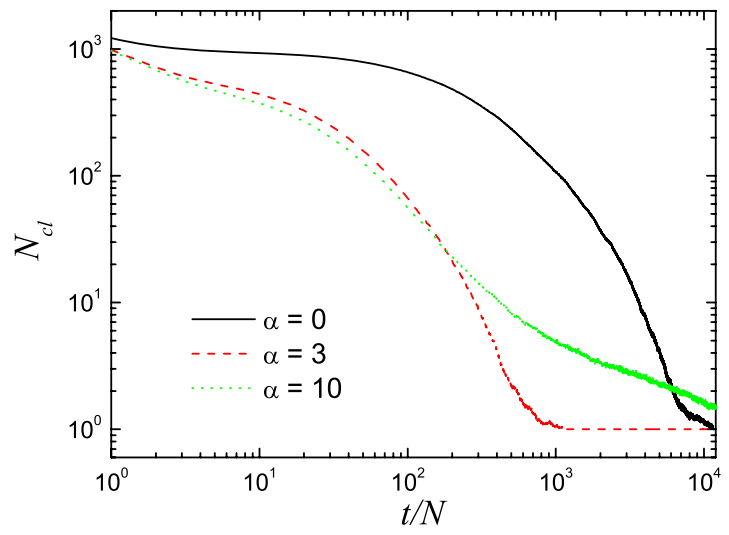

FIG. 5. (Color online) The number of opinion clusters $N_{c l}$ versus rescaled time $t / N$ for different values of $\alpha$. The average connectivity of the BA network $\langle k\rangle=6$, the network size $N=2000$ and the number of initial opinions $G=N=2000$. Each data point is obtained by averaging over 50 different network realizations with 50 runs for each realization.

distribution affects the modified VM, we consider the growing network with redirection (GNR) [41]. The GNR is built by adding nodes sequentially, where each new node attaches either to a randomly selected node with probability $1-r$ or to the ancestor of this target with probability $r$. We chose the out degree of each node to be two, and redirection was applied to each outgoing link of the new node. The GNR has a power-law degree distribution $n_{k} \propto k^{-\gamma}$, with the exponent $\gamma$ $=1+1 / r$ in the range $(2, \infty)$ as $r$ is varied between 0 and 1 .

From Fig. 7, one can observe that there still exists an optimal value of $\alpha$, leading to the shortest consensus time on the GNR. As the exponent of the power-law degree distribution $\gamma$ increases, the network becomes more homogeneous and needs higher value of $\alpha$ to provide enough influence for high-degree nodes. Thus the optimal value of $\alpha$ increases as the exponent $\gamma$ increases.

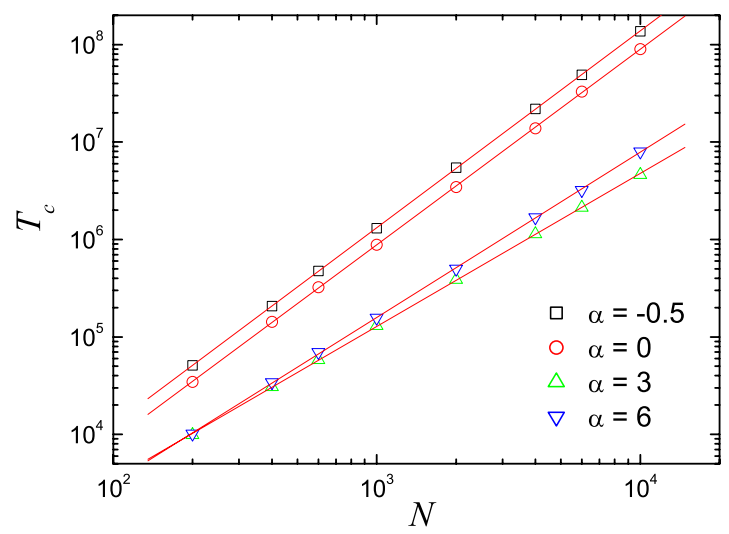

FIG. 6. (Color online) Consensus time $T_{c}$ versus the network size $N$ for different values of $\alpha$. The average connectivity of the BA network $\langle k\rangle=6$ and the number of initial opinions $G=2 . T_{c}$ scales as $N^{\beta}, \quad \beta=2.02,2.00,1.57,1.69$, respectively, correspond to $\alpha=$ $-0.5,0,3,6$. Each data point is obtained by averaging over 50 different network realizations with 50 runs for each realization. 


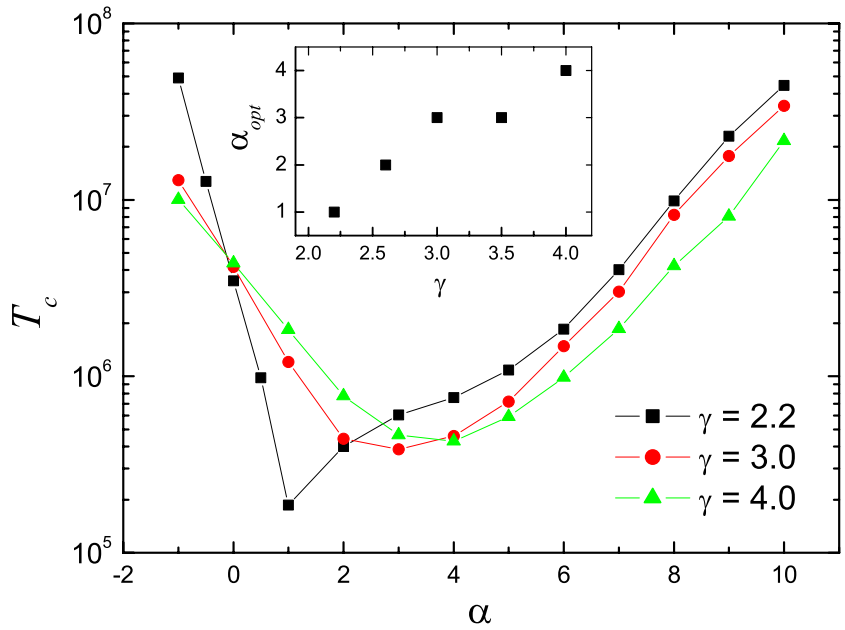

FIG. 7. (Color online) Consensus time $T_{c}$ versus $\alpha$ on GNR networks with degree distribution $n_{k} \propto k^{-\gamma}$ for $\gamma=2.2,3.0$, and 4.0. The inset shows the optimal values $\alpha_{\text {opt }}$ as a function of $\gamma$. The average connectivity $\langle k\rangle=4$, the network size $N=2000$ and the number of initial opinions $G=2$. Each data point is obtained by averaging over 50 different network realizations with 50 runs for each realization.

\section{CONCLUSIONS}

In conclusion, we have studied a variant of the voter model with respect to the social diversity on scale-free networks. The diversity of individuals is realized by assigning a weight - which represents the strength of social influenceto each individual, and the probability of choosing the opinion of one of two selected individuals as their common opinion is proportional to this individual's weight. A tunable parameter $\alpha$ is introduced to govern the weight based on the degree of individuals. The most interesting result is that there exists an optimal value of the parameter $\alpha$ that leads to the fastest consensus. The optimal value of $\alpha$ increases as the average connectivity and the exponent of the power-law degree distribution increase while is almost independent of the network size and the number of initial opinions. These results demonstrate that a proper proportion of the individuals' influential weights to their interaction degrees can best benefit the achievement of global consensus, and high-degree individuals may play both positive (being properly assigned higher weights) and negative (being assigned over weights) roles in the opinion dynamics. We have qualitatively explained such nonmonotonic behavior in terms of the probability of an individual whose initial opinion becomes the final opinion as a function of degree and the evolution of the number of opinion clusters. Furthermore, we have investigated the scaling behavior of the consensus time with the network size. Since the social diversity is widely existed in the human society and the animal world, our work may be of practical significance.

Note Added. Recently, the referee pointed to us that a very similar extension of the voter model has been proposed independently in a recent work [42]. In this paper, SchneiderMizell and Sander mainly studied the scaling of consensus time. In our work, we find that there exists an optimal value of $\alpha$, leading to the shortest consensus time. We also study how the optimal value of $\alpha$ changes with different network topologies. All together Ref. [42] and our work have provided a more comprehensive understanding of the impact of social diversity on opinion dynamics.

\section{ACKNOWLEDGMENTS}

We thanks Wen-Xu Wang for useful discussions. This work was funded by the National Basic Research Program of China (973 Program No. 2006CB705500), the National Natural Science Foundation of China (Grants No. 10975126, No. 60744003, No. 10635040, and No. 10532060), the Specialized Research Fund for the Doctoral Program of Higher Education of China (Grant No. 20060358065), and the National Science Fund for Fostering Talents in Basic Science (Grant No. J0630319). Z.X.W. acknowledges the support from the Swedish Research Council. C.S.Z. was supported by Hong Kong Baptist University.
[1] K. Sznajd-Weron and J. Sznajd, Int. J. Mod. Phys. C 11, 1157 (2000).

[2] S. Galam, Eur. Phys. J. B 25, 403 (2002).

[3] D. J. Watts, Proc. Natl. Acad. Sci. U.S.A. 99, 5766 (2002).

[4] S. Galam, Physica A 320, 571 (2003).

[5] R. Pastor-Satorras and A. Vespignani, Phys. Rev. Lett. 86, 3200 (2001).

[6] T. Zhou, Z.-Q. Fu, and B.-H. Wang, Prog. Nat. Sci. 16, 452 (2006).

[7] L. Dall'Asta, A. Baronchelli, A. Barrat, and V. Loreto, Phys. Rev. E 74, 036105 (2006).

[8] C. Castellano, S. Fortunato, and V. Loreto, Rev. Mod. Phys. 81, 591 (2009).

[9] S. Galam, Europhys. Lett. 70, 705 (2005).

[10] S. Galam, Physica A 274, 132 (1999).

[11] P. L. Krapivsky and S. Redner, Phys. Rev. Lett. 90, 238701
(2003).

[12] S. Galam and F. Jacobs, Physica A 381, 366 (2007).

[13] T. M. Liggett, Interacting Particle Systems (Springer-Verlag, New York, 1985).

[14] G. Duffuant, F. Amblard, G. Weisbuch, and T. Faure, J. Artif. Soc. Soc. Simul. 5, 4 (2002).

[15] R. Albert and A.-L. Barabási, Rev. Mod. Phys. 74, 47 (2002).

[16] M. E. J. Newman, SIAM Rev. 45, 167 (2003).

[17] A. D. Sánchez, J. M. López, and M. A. Rodríguez, Phys. Rev. Lett. 88, 048701 (2002).

[18] S. Galam, Physica A 333, 453 (2004).

[19] P. Chen and S. Redner, Phys. Rev. E 71, 036101 (2005).

[20] C. Castellano, V. Loreto, A. Barrat, F. Cecconi, and D. Parisi, Phys. Rev. E 71, 066107 (2005)

[21] V. Sood and S. Redner, Phys. Rev. Lett. 94, 178701 (2005).

[22] K. Suchecki, V. M. Eguíluz, and M. S. Miguel, Phys. Rev. E 
72, 036132 (2005).

[23] L. F. C. Pereira and F. G. Brady Moreira, Phys. Rev. E 71, 016123 (2005).

[24] P. Holme and M. E. J. Newman, Phys. Rev. E 74, 056108 (2006).

[25] M. C. González, A. O. Sousa, and H. J. Herrmann, Eur. Phys. J. B 49, 253 (2006).

[26] P.-P. Li, D.-F. Zheng, and P. M. Hui, Phys. Rev. E 73, 056128 (2006).

[27] R. Lambiotte, EPL 78, 68002 (2007).

[28] R. Lambiotte, M. Ausloos, and J. A. Hołyst, Phys. Rev. E 75, 030101(R) (2007).

[29] V. Sood, T. Antal, and S. Redner, Phys. Rev. E 77, 041121 (2008).

[30] H.-U. Stark, C. J. Tessone, and F. Schweitzer, Phys. Rev. Lett. 101, 018701 (2008).

[31] B. Kozma and A. Barrat, Phys. Rev. E 77, 016102 (2008).

[32] L.-L. Jiang, D.-Y. Hua, J.-F. Zhu, B.-H. Wang, and T. Zhou,
Eur. Phys. J. B 65, 251 (2008).

[33] F. Fu and L. Wang, Phys. Rev. E 78, 016104 (2008).

[34] J. Shao, S. Havlin, and H. E. Stanley, Phys. Rev. Lett. 103, 018701 (2009).

[35] S. Galam, Phys. Rev. E 71, 046123 (2005).

[36] J.-Y. Guan, Z.-X. Wu, and Y.-H. Wang, Phys. Rev. E 76, 042102 (2007).

[37] M. Perc and A. Szolnoki, Phys. Rev. E 77, 011904 (2008).

[38] F. C. Santos, M. D. Santos, and J. M. Pacheco, Nature (London) 454, 213 (2008).

[39] H.-X. Yang, W.-X. Wang, Z.-X. Wu, Y.-C. Lai, and B.-H. Wang, Phys. Rev. E 79, 056107 (2009).

[40] A.-L. Barabási and R. Albert, Science 286, 509 (1999).

[41] P. L. Krapivsky and S. Redner, Phys. Rev. E 63, 066123 (2001).

[42] C. M. Schneider-Mizell and L. M. Sander, J. Stat. Phys. 136, 59 (2009). 